\title{
Malignant intertrigo
}

\author{
Connie Liu MD, Mei-Ju Ko MD PhD
}

Cite as: CMAJ 2018 May 14;190:E595. doi: 10.1503/cmaj.180078

A 55-year-old woman was referred to the dermatology department for an intertriginous eruption of one week's duration. She had undergone breastconserving surgery for stage 2 breast cancer. Two weeks before the start of the eruption, she had been started on a chemotherapy regimen with cyclophosphamide and doxorubicin.

On physical examination, the patient had multiple painful erythematous to dull-red patches with crusting and erosions over the intertriginous areas including the axillae and groin (Figure 1A). Scattered bullae were also found in the axillary region (Figure 1B). Skin cultures were taken to test for the presence of bacteria and fungi; results for these tests were negative.

A biopsy of the right axilla showed subepidermal and suprabasal vesicular dermatitis with epidermal necrosis and mixed infiltration of neutrophils and eosinophils. These findings were consistent with malignant intertrigo, the intertriginous pattern of toxic erythema caused by chemotherapy. The eruption responded well to prednisolone administered orally $(5 \mathrm{mg}$ twice daily), along with topical betamethasone and zinc oxide suspension (twice daily).

Malignant intertrigo often presents with erythematous patches with scales, crusts and erosions over intertriginous regions that usually develops two days to three weeks after the administration of chemotherapeutic agents. ${ }^{1}$ Its pathophysiology is presumed to be caused by local accumulation of cytotoxic chemicals. At present, cytarabine and pegylated liposomal doxorubicin are the most frequently reported causative agents. ${ }^{1,2}$ This condition is usually a selflimiting eruption; however, recurrence with readministration of the offending agent is not uncommon. ${ }^{1,2}$

Usually, malignant intertrigo is not reported, and the incidence may be higher than expected. Diagnosis is based mainly on typical clinical presentation. ${ }^{1,2}$ Negative results for bacterial and fungal cultures, and no satellite lesions at the periphery distinguish malignant intertrigo from infectious intertrigo. Intertriginous distribution discriminates malignant intertrigo from other chemotherapy-related skin eruptions. Early recognition of this entity, and management with topical or oral cortico- steroids and general topical measures can provide palliation of symptoms, and prevent unnecessary and potentially harmful treatments. ${ }^{1,3}$

\section{References}

1. Smith SM, Milam P, Fabbro S, et al. Malignant intertrigo: a subset of toxic erythema of chemotherapy requiring recognition. JAAD Case Rep 2016;2:476-81.

2. Martorell-Calatayud A, Sanmartín O, Botella-Estrada R, et al. Chemotherapyrelated bilateral dermatitis associated with eccrine squamous syringometaplasia: reappraisal of epidemiological, clinical, and pathological features. J Am Acad Dermatol 2011;64:1092-103.

3. Carvalho R, Macias V, Marques-Pinto G, et al. Intertriginous pattern of toxic erythema of chemotherapy. Cutan Ocul Toxicol 2011;30:309-11.

\section{Competing interests: None declared.}

This article has been peer reviewed.

The authors have obtained patient consent.

Affiliations: Department of Dermatology (Liu, Ko), Taipei City Hospital; Department of Dermatology (Ko), National Taiwan University Hospital and College of Medicine, Taipei, Taiwan

Correspondence to: Mei-Ju Ko, kmj@ntu.edu.tw

Clinical images are chosen because they are particularly intriguing, classic or dramatic. Submissions of clear, appropriately labelled highresolution images must be accompanied by a figure caption. A brief explanation (300 words maximum) of the educational importance of the images with minimal references is required. The patient's written consent for publication must be obtained before submission. 